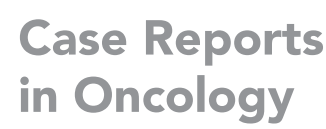

\title{
Distant Metastasis after Surgery for Encapsulated Papillary Carcinoma of the Breast: A Case Report
}

\author{
Miyuki Kitahara ${ }^{a}$ Yasuo Hozumi ${ }^{a}$ Naoto Takeuchia Satoko Ichinohe ${ }^{a}$ \\ Saori Fujiwara $^{a}$ Mitsuki Machinaga $^{a}$ Hitoaki Saitoh $^{b}$ Tatsuo lijima $^{b}$ \\ aDepartment of Breast Surgery, Ibaraki Prefectural Central Hospital and Ibaraki Cancer \\ Center, Kasama, Japan; b Department of Diagnostic Pathology, Ibaraki Prefectural Central \\ Hospital and Ibaraki Cancer Center, Kasama, Japan
}

\author{
Keywords \\ Ductal carcinoma in situ · Breast cancer · Distant metastasis · Encapsulated papillary \\ carcinoma
}

\begin{abstract}
In the absence of clear interstitial invasion, encapsulated papillary carcinoma (EPC) of the breast may be attributed to an extremely good prognosis if handled similarly to ductal carcinoma in situ (DCIS) with suitable local treatment. Here, we report our experience with a case of EPC of the breast that presented with carcinomatous pleuritis and lymphangitis carcinomatosa postoperatively, which rapidly resulted in a poor outcome. A 67-year-old woman was diagnosed with DCIS of the left breast and underwent left partial mastectomy and sentinel lymph node biopsy. EPC was diagnosed because the pathological examination showed no sign of interstitial infiltration. Postoperative radiation therapy was performed. Five years and 9 months postoperatively, the patient began experiencing cough and shortness of breath on exertion. Imaging showed right pleural effusion and consolidation of the lung field, but nothing suggesting local recurrence in the preserved left breast, local lymph nodes, or opposite breast was observed. Postoperative recurrence of breast cancer, carcinomatous pleuritis, and lymphangitis carcinomatosa were diagnosed based on the results of pleural fluid cytology. One month later, multiple brain metastases were found, and the patient died of the primary disease 5 months after recurrence. After surgery for EPC without clear interstitial infiltration, there was a small possibility of a poor outcome from distant metastasis. Therefore, although distant metastasis is uncommon, regular examination and testing should be performed.
\end{abstract}

\section{Karger $\stackrel{2}{*}$}




\section{Case Reports in Oncology}

\begin{tabular}{l|l}
\hline Case Rep Oncol 2020;13:1196-1201 \\
\hline DOI: 10.1159/000510308 & $\begin{array}{l}\text { ○ 2020 The Author(s). Published by S. Karger AG, Basel } \\
\text { www.karger.com/cro }\end{array}$ \\
\hline
\end{tabular}

Kitahara et al.: Postoperative Distant Metastasis in EPC of the Breast

\section{Introduction}

Encapsulated papillary carcinoma (EPC) of the breast is a new disease concept proposed in the 4th edition of the World Health Organization (WHO) classification in 2012 [1]. In the 5 th edition in 2019, it is listed as a papillary neoplasm under epithelial tumors [2]. EPC is a rare breast disease, accounting for approximately $0.5-2 \%$ of all breast cancers [3]. It is recommended that EPC cases without clear interstitial invasion be treated similarly to ductal carcinoma in situ (DCIS), and if appropriate local treatment is provided, the prognosis is extremely good [2]. Here, we report our experience with a case of EPC of the breast that presented with carcinomatous pleuritis and lymphangitis carcinomatosa postoperatively, which rapidly resulted in a poor outcome.

\section{Case Report}

A 67-year-old woman underwent left partial mastectomy and sentinel lymph node biopsy for DCIS in the upper-medial left breast. She had no pertinent past family and psychosocial medical history, including relevant genetic information. Upon histopathological examination, papillary, cribriform ductal structures inside dilated ducts and solid proliferating tumor tissue were seen, but no signs of infiltrative changes or vascular invasion around the ducts. Immunohistochemical staining for p63 and cytokeratin (CK) 5/6 showed a mixture of areas

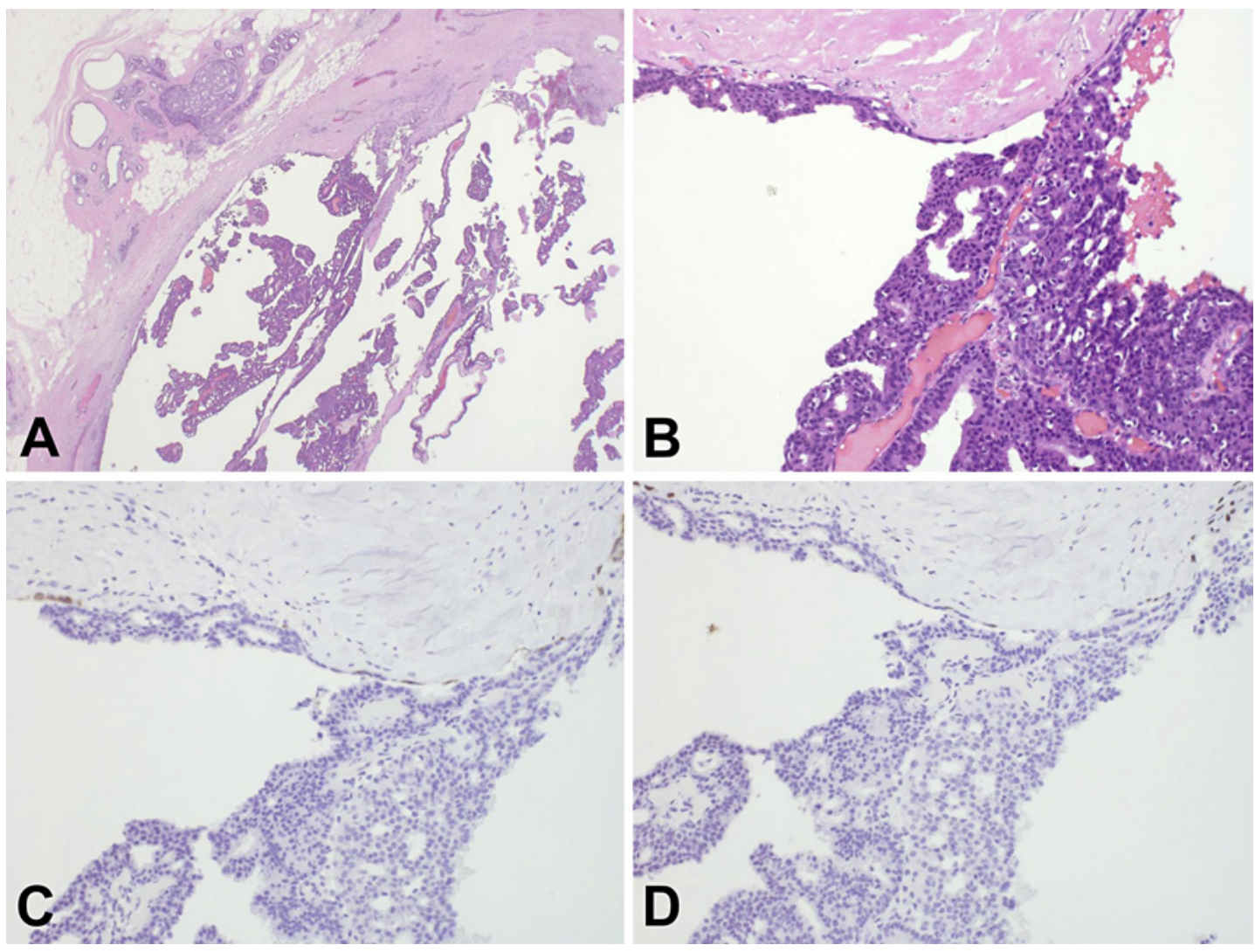

Fig. 1. Histopathological findings in the resected specimen. A Tumor tissue with papillary proliferation inside the dilated ducts $(\mathrm{HE}, \times 20)$. B HE, $\times 200$. C Immunohistochemical staining $(\mathrm{CK5} / 6)$ showing a mixture of areas with ducts with two-layer structures and areas where these structures have disappeared $(\times 200)$. D p63, ×200. HE, hematoxylin eosin; CK5/6, cytokeratin 5/6. 

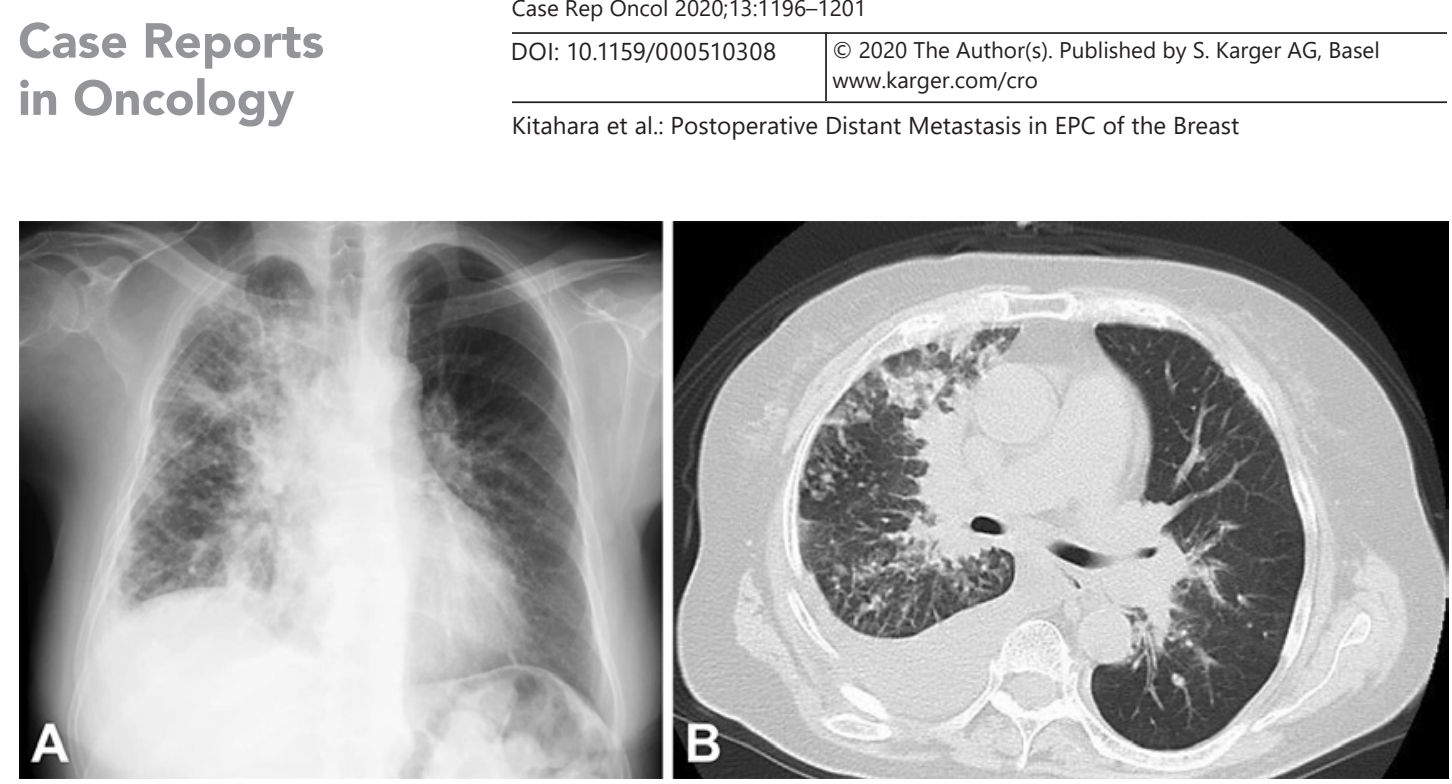

Fig. 2. Imaging taken when the patient's respiratory symptoms indicated right pleural effusion and diffuse consolidation in the lung field. A Chest radiography. B Chest CT. CT, computed tomography.

where ducts had a two-layer structure and areas where this structure had disappeared (Fig. 1). Based on these histopathological findings, we diagnosed the lesion as EPC without interstitial invasion. The tumor was $29 \times 18 \times 60 \mathrm{~mm}$ in size, with a nuclear grade of 1 , and had neither lymphatic nor venous invasion. The lesion was estrogen receptor (ER) and progesterone receptor (PgR) positive, and the Ki-67 labeling index was 6\%. The resection margin was positive on the nipple and lateral sides, and there was no metastasis to the four sentinel lymph nodes. The Tumor-Node-Metastasis (TNM) classification was pTis (DCIS) NOM0. The patient's prognosis appeared favorable based on the histopathological findings, and radiation therapy was performed on the left breast and tumor bed after surgery.

Five years and 9 months postoperatively, the patient began experiencing cough and shortness of breath on exertion. Chest radiography and computed tomography revealed right pleural effusion and diffuse consolidation in the lung field (Fig. 2). Nothing suggesting local recurrence was observed in the preserved left breast, local lymph nodes, or opposite breast. Imaging showed nothing abnormal in other organs, but adenocarcinoma cells were found in the pleural fluid cytology (Fig. 3A, B). Immunohistochemical staining indicated that the cells were positive for ER, PgR (Fig. 3C, D), and CK7, and negative for CK20, gross cystic disease fluid protein 15, thyroid transcription factor 1, napsin A, SP-A, calretinin, desmin, and p53. Based on these results, we diagnosed recurrence of breast cancer with carcinomatous pleuritis and lymphangitis carcinomatosa. Chemotherapy was proposed for systemic pharmacotherapy, but the patient refused. Hormone therapy with oral letrozole was instead initiated. Multiple brain metastases were found 1 month later after the patient had fallen repeatedly. The treatment plan was switched to best supportive care. Five months after the recurrence and 6 years and 2 months after the operation, the patient died of the primary disease.

\section{Discussion}

EPC is a rare breast disease [3], traditionally classified as a noninvasive form of breast cancer and a variant subtype of DCIS. Clinically, it often presents as a round breast mass with clear boundaries in postmenopausal women and may be accompanied by abnormal nipple discharge. EPC exhibits imaging findings similar to those of other papillary tumors, such as intraductal papilloma and DCIS. Thus, it is often difficult to diagnose this disease based on

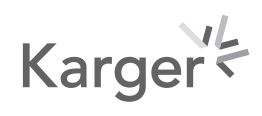




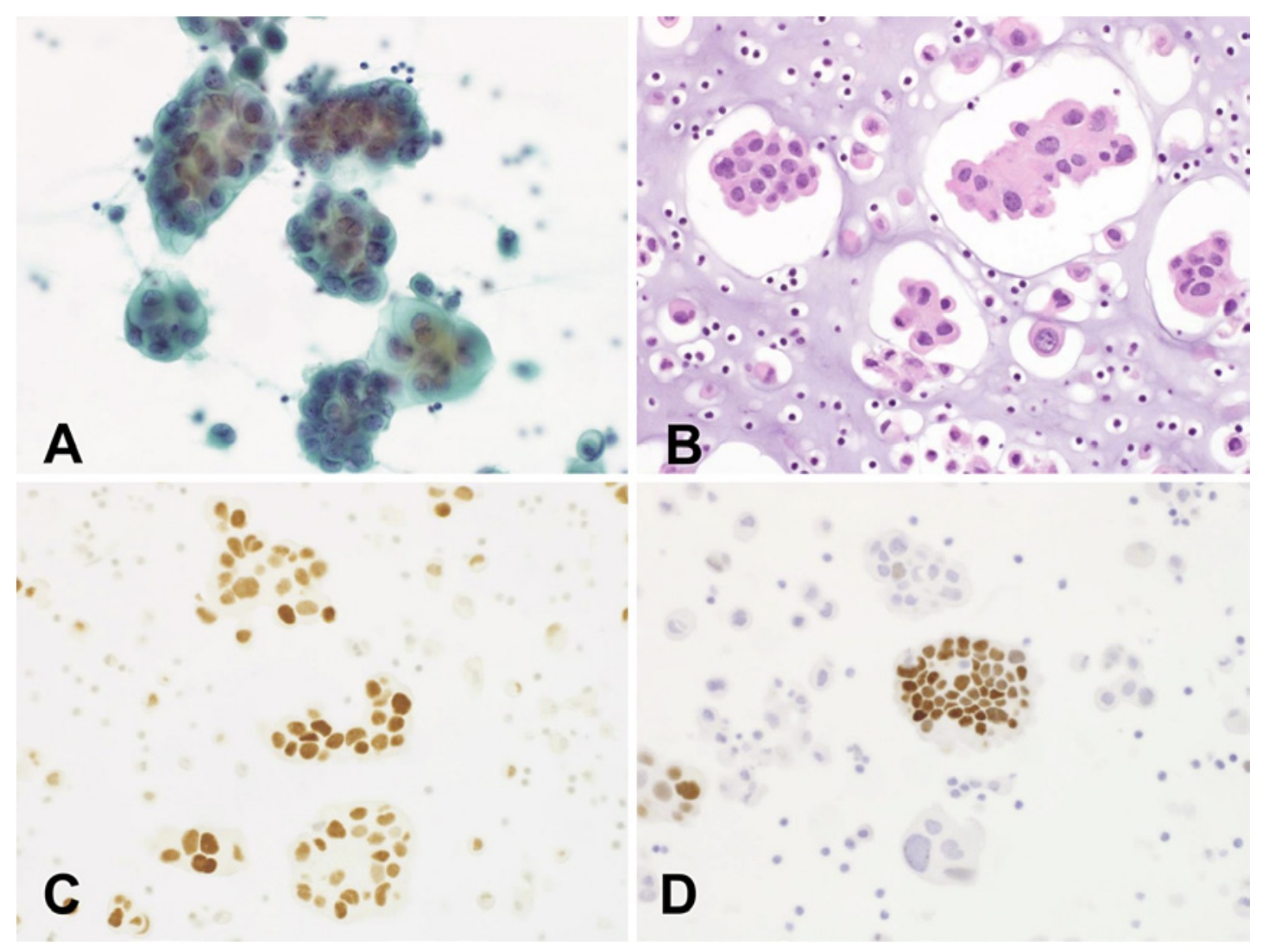

Fig. 3. Pleural fluid cytology findings. A Papanicolaou staining showing numerous conglomerations of adenocarcinoma cells $(\times 400)$. B Cell block specimens showing numerous conglomerations exhibiting pyknosis, anisokaryosis, and irregular nuclear shapes. Adenocarcinoma was diagnosed based on these findings (HE, $\times 400)$. C Estrogen receptor staining $(\times 400)$. D Progesterone receptor staining $(\times 400)$. HE, hematoxylin eosin.

clinical findings alone [2]. In the present case, the lesion was originally diagnosed as DCIS based on a needle biopsy of the tumor. Diagnosing the presence or absence of invasion and the detailed histology proved to be difficult.

Macroscopically, EPC often presents as a round cystic tumor with a clear boundary and expansive growth. Histopathologically, EPC is composed of multiple fibrous vascular pedicles covered with homogeneous epithelial tumor cells with low-to-moderate dyskaryosis, and surrounded by a capsule. Normally, myoepithelial cells are not found in the interstitium of the vascular pedicles and the tumor cells, although immunohistochemistry for myoepithelial markers (p63, calponin, CD10, etc.) sometimes confirms a small number of myoepithelial cells on the tumor margin [2].

In EPC, infiltrative growth of tumor cells beyond the capsule into the surrounding tissue coating is defined as a "frank invasion." This is also often seen in regular invasive ductal carcinoma [2]. However, the present case did not exhibit clear interstitial invasion. The working group for the 4th edition of the WHO classification recommended that, to avoid overtreatment, EPC without clear interstitial invasion be handled similarly to DCIS and evaluated as pTis (DCIS) in the TNM stage classification [1]. EPC without clear interstitial invasion beyond the capsule may have a very good prognosis if appropriate local treatment is provided. However, in rare cases, EPC with no myoepithelial cells in the tumor margin can develop axillary lymph node and distant metastasis [3]. Therefore, in recent years, EPC without clear 
interstitial invasion has been considered a self-confined indolent invasive carcinoma that has slow local growth and a prognosis similar to that of noninvasive carcinoma [2].

Currently, surgery is the standard treatment for DCIS. Further, it is customary to add axial lymph node dissection to radiation therapy of the breast after partial mastectomy if a sentinel lymph node biopsy is positive or if lymph node metastasis is found during the mastectomy. When a partial mastectomy is performed, internal endocrine therapy is often administered to prevent recurrence in the breast on that side. Endocrine therapy is selected when there is no clear reason for breast irradiation or when there is thought to be a high risk of recurrence due to a positive resection margin or high nuclear grade [4-7].

The prognosis of DCIS is extremely good, and lymph node and distant metastases are rare. The 10-year breast cancer-specific survival rate is reported to be 98\% [8]. Distant metastasis after DCIS surgery is reported to occur in less than $1 \%$ of cases $[9,10]$. Many cases of DCIS that develop postoperative distant metastasis also exhibit recurrence locally or in the opposite breast before or simultaneously to the distant metastasis. The US National Surgical Adjuvant Breast Project B-17 compared patients after partial mastectomy with and without breast irradiation. Out of 814 DCIS cases, only $6(0.74 \%)$ exhibited distant metastasis as the first recurrence [11].

Factors affecting distant metastasis after surgery for DCIS include young age ( $\leq 40$ years), lymph node metastasis, microinvasion, comedo necrosis, ER negativity, poor differentiation, and previous or simultaneous local recurrence $[10,12,13]$. In addition, human epidermal growth factor receptor type 2 positivity and a high Ki-67 labeling index $(>10 \%)$ have been reported to be pathological prognostic markers associated with recurrence and distant metastasis $[14,15]$. However, due to the small number of cases of distant metastasis after surgery for DCIS, these previously reported factors have not yet been shown to be statistically significant. The present case did not have any of these factors affecting distant metastasis. There was no local recurrence in the present case in the preserved breast, opposite breast, or local lymph nodes. In the present case, the tumor was a large ductal lesion with a diameter of $60 \mathrm{~mm}$ and a positive resection margin. However, we cannot rule out the possibility of some invasive carcinoma with tiny infiltrations that we have missed in the resected specimen, images of the preserved breast, or pathological diagnosis. If it is thought cancer cells might remain in the preserved breast and the risk of recurrence is a concern, additional mastectomy and endocrine therapy should be considered.

The prognosis of EPC of the breast without interstitial invasion is generally as good as that of DCIS. However, in rare cases, distant metastasis may occur after surgery, which leads to a poor outcome. Therefore, although distant metastasis is uncommon, regular examination and testing should be performed.

\section{Acknowledgments}

The authors would like to thank the patient's family for providing consent to publish the patient's clinical information and data. We would like to thank Editage (www.editage.com) for English language editing.

\section{Statement of Ethics}

The patient's family provided written informed consent to publish her case (including publication of images).

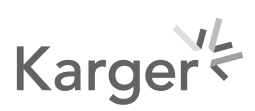




\section{Conflict of Interest Statement}

The authors have no conflicts of interest to declare.

\section{Funding Sources}

No funding was received.

\section{Author Contributions}

M.K. drafted the work. Y.H., N.T., S.I., S.F., M.M., H.S., and T.I. substantively revised it. All authors read and approved the final manuscript.

\section{References}

1 Lekhani SR, Ellis IO, Schnitt SJ, Tan PH, van de Vijver MJ. WHO classification of tumours of the breast. 4th ed. Lyon: IARC Press; 2012.

2 WHO Classification of Tumours Editorial Board. WHO classification of tumours: breast tumours. 5th ed. Lyon: IARC Press; 2019.

3 Fayanju OM, Ritter J, Gillanders WE, Eberlein TJ, Dietz JR, Aft R, et al. Therapeutic management of intracystic papillary carcinoma of the breast: the roles of radiation and endocrine therapy. Am J Surg. 2007 Oct;194(4): 497-500.

4 National Comprehensive Cancer Network. NCCN guidelines: NCCN clinical practice guidelines in oncology v3. Breast Cancer [Internet]. [cited 2019 Sep 6]. https://www.nccn.org/professionals/physician_gls/default. aspx.

5 Inokuchi M, Kutomi G, Kijima Y, Sakai T, Sawaki M, Shien T, et al. The Japanese Breast Cancer Society clinical practice guidelines for surgical treatment of breast cancer, 2018 edition. Breast Cancer. 2020 Jan;27(1):4-8.

6 Yamauchi C, Sekiguchi K, Nishioka A, Arahira S, Yoshimura M, Ogo E, et al. The Japanese Breast Cancer Society Clinical Practice Guideline for radiation treatment of breast cancer, 2015 edition. Breast Cancer. 2020 Jan; 23(3):378-90.

7 Shimoi T, Nagai SE, Yoshinami T, Takahashi M, Arioka H, Ishihara M, et al. The Japanese Breast Cancer Society Clinical Practice Guidelines for systemic treatment of breast cancer, 2018 edition. Breast Cancer. 2020 May; 27(3):322-31.

8 Wapnir IL, Dignam JJ, Fisher B, Mamounas EP, Anderson SJ, Julian TB, et al. Long-term outcomes of invasive ipsilateral breast tumor recurrences after lumpectomy in NSABP B-17 and B-24 randomized clinical trials for DCIS. J Natl Cancer Inst. 2011 Mar;103(6):478-88.

9 Meijnen P, Oldenburg HS, Peterse JL, Bartelink H, Rutgers EJ. Clinical outcome after selective treatment of patients diagnosed with ductal carcinoma in situ of the breast. Ann Surg Oncol. 2008 Jan;15(1):235-43.

10 Roses RE, Arun BK, Lari SA, Mittendorf EA, Lucci A, Hunt KK, et al. Ductal carcinoma-in-situ of the breast with subsequent distant metastasis and death. Ann Surg Oncol. 2011 Oct;18(10):2873-8.

11 Fisher B, Dignam J, Wolmark N, Mamounas E, Costantino J, Poller W, et al. Lumpectomy and radiation therapy for the treatment of intraductal breast cancer: findings from National Surgical Adjuvant Breast and Bowel Project B-17. J Clin Oncol. 1998 Feb;16(2):441-52.

12 Yen TW, Hunt KK, Ross MI, Mirza NQ, Babiera GV, Meric-Bernstam F, et al. Predictors of invasive breast cancer in patients with an initial diagnosis of ductal carcinoma in situ: a guide to selective use of sentinel lymph node biopsy in management of ductal carcinoma in situ. J Am Coll Surg. 2005 Apr;200(4):516-26.

13 Bijker N, Peterse JL, Duchateau L, Julien JP, Fentiman IS, Duval C, et al. Risk factors for recurrence and metastasis after breast-conserving therapy for ductal carcinoma-in-situ: analysis of European Organization for Research and Treatment of Cancer trial 10853. J Clin Oncol. 2001 Apr;19(8):2263-71.

14 Rakovitch E, Nofech-Mozes S, Hanna W, Narod S, Thiruchelvam D, Saskin R, et al. HER2/neu and Ki-67 expression predict non-invasive recurrence following breast-conserving therapy for ductal carcinoma in situ. Br J Cancer. 2012 Mar; 106(6):1160-5.

15 Davis JE, Nemesure B, Mehmood S, Nayi V, Burke S, Brzostek SR, et al. Her2 and Ki67 biomarkers predict recurrence of ductal carcinoma in situ. Appl Immunohistochem Mol Morphol. 2016 Jan;24(1):20-5. 